\title{
Prevalence and Risk Factors of Developing Fistula or Organ Perforation in Patients Treated with Lenvatinib for Radioiodine-Refractory Thyroid Cancer
}

\author{
Laura Valerio $^{a}$ Carlotta Giani $^{a} \quad$ Laura Agate $^{a} \quad$ Eleonora Molinaro $^{a}$ \\ David Viola ${ }^{a}$ Valeria Bottici ${ }^{a}$ Antonio Matrone ${ }^{a}$ Luciana Puleo $^{a}$ \\ Loredana Lorusso $^{a}$ Virginia Cappaglia Alessandro Ribechini ${ }^{b}$ Rossella Elisei $^{a}$ \\ aDepartment of Clinical and Experimental Medicine, Unit of Endocrinology, University Hospital of Pisa, Pisa, Italy; \\ bepartment of Thoracic Endoscopy, University Hospital of Pisa, Pisa, Italy
}

\section{Keywords}

Lenvatinib · Thyroid cancer · Radioiodine-refractory

thyroid cancer - Tyrosine kinase inhibitors - Adverse events · Fistula/organ perforation

\begin{abstract}
Introduction: Tyrosine kinase inhibitors represent a better treatment in patients with radioiodine-refractory differentiated thyroid cancer (RAI-R DTC). Lenvatinib is usually welltolerated, but sometimes, it is associated with serious and even life-threatening side effects. The aim of this study was to evaluate the prevalence of and the potential risk factors for fistula and/or organ perforation in RAI-R DTC patients treated with lenvatinib. Methods: This study included data from advanced and progressive RAI-R DTC patients treated with lenvatinib from February 2011 to February 2020 who were followed up at a single center. The clinical-pathological features and the biochemical and morphological results of the patients were collected at the time of starting lenvatinib and during the follow-up. Results: Fourteen of 95 (14.7\%) locally advanced or metastatic RAI-R DTC patients treated with lenvatinib developed a fistula or organ perforation. Nine of
\end{abstract}

karger@karger.com www.karger.com/et

C 2021 European Thyroid Association Published by S. Karger AG, Basel
$14(64.3 \%)$ patients had tumor infiltration of the trachea, bronchus, esophagus, pleura, or bladder. Five of 14 (35.7\%) had a bowel perforation, but only 2 had preexisting diverticulosis. Evaluation of the risk factors for developing a fistula or organ perforation showed that the presence of tumor infiltration and the tumor histology (papillary and poorly differentiated vs. follicular and Hurthle thyroid cancer) were significantly correlated with the development of a fistula or organ perforation ( $p=0.003$ and $p=0.02$, respectively). In the subgroup of patients with tumor infiltration, we found that the papillary thyroid cancer histotype was the only potential predictor of fistula development. External beam radiation therapy (EBRT), the starting dose of lenvatinib, and the duration of treatment were not relevant for the development of fistula. Conclusions: In metastatic thyroid cancer patients treated with lenvatinib, the presence of tumor infiltration and histological type should be considered as potential risk factors for the development of fistula or organ perforation, although they do not represent an absolute contraindication. Although EBRT and the presence of diverticulosis

Laura Valerio and Carlotta Giani contributed equally to this study. 
were not significantly associated with the development of fistula and organ perforation, they should be regarded as potential additional reasons for the development of these complications. According to our findings, there is no reason to start lenvatinib at a lower daily dose when tumor infiltration is present.

(c) 2021 European Thyroid Association Published by S. Karger AG, Basel

\section{Introduction}

Tyrosine kinase inhibitors (TKIs) are the standard of care for patients with advanced and progressive radioiodine-refractory differentiated thyroid cancer (RAI-R DTC) [1]. In particular, both sorafenib and lenvatinib have been approved for the treatment of these patients based on the results of phase III studies (DECISION and SELECT studies) $[2,3]$.

Like many other TKIs, lenvatinib is a multiple kinase inhibitor and inhibits the 3 main vascular endothelial growth factor receptors (VEGFRs 1, 2, 3), the plateletderived growth factor receptor (PDGFR), RET, and the $c$-KIT proto-oncogene. Lenvatinib is usually well-tolerated, but serious and even life-threatening side effects have been correlated with its use. Sporadic cases of aerodigestive fistula and intestinal perforation in patients on TKIs treatment have been reported [4-8]. In the SELECT study, fistula or gastrointestinal perforation was observed in $2 \%$ of patients treated with lenvatinib compared to $0.8 \%$ in the placebo group [9].

Although the mechanism(s) responsible for these severe complications is still unknown, it is thought that the very strong action against VEGFR, and in particular against VEGFR-2 $\left(\mathrm{IC}_{50}=4 \mathrm{nM}\right)[10]$, could be the main reason for side effects such hypertension, fistula, and tumor bleeding [11]. This hypothesis is supported by the evidence that intestinal perforations affect approximately $5 \%$ of patients with other cancers treated with bevacizumab, a humanized monoclonal VEGF antibody $[12,13]$. A few cases of intestinal perforation have been reported in patients with other cancers treated with TKIs $[12,13]$, but it is worth noting that the majority of them had a history of endoscopy or abdominal radiotherapy, abdominal carcinomatosis, or diverticulitis, which might represent possible risk factors [12, 13].

The aim of this study was to evaluate the prevalence of fistulas and/or organ perforation in a large series of patients treated with lenvatinib for RAI-R DTCs. An analysis of the potential risk factors for these severe adverse events (AEs) was performed.

\section{Materials and Methods}

\section{Patients}

This retrospective study included data from RAI-R DTC patients treated with lenvatinib from February 2011 to February 2020 and followed up at the Endocrine Unit of the University Hospital of Pisa. Our patients were treated with lenvatinib within clinical trials or as "compassionate use" or with the commercial drug after its approval. The lenvatinib clinical trials were part of the SELECT study - A multicenter, randomized, double-blind, placebo-controlled, phase 3 trial of lenvatinib (E7080) in 131I-refractory differentiated thyroid cancer (NCT01321554) [2] and the expanded access program (EAP) study [14].

All patients were adults with locally advanced or metastatic RAI-R DTC or poorly differentiated thyroid cancer (PDTC). Patients enrolled in clinical trials had to have satisfied the enrolling criteria of the specific clinical trials $[2,14]$. For enrollment in those trials, patients must have a RAI-R DTC and radiographic disease progression determined by the modified Response Evaluation Criteria in Solid Tumors (RECIST) guidelines at screening [15]. In these clinical trials, significant cardiac, hematopoietic, hepatic, and renal failure were considered to be exclusion criteria.

Patients who were treated with lenvatinib as a "compassionate use" or after its approval started the treatment either because of evidence of progression or the presence of a large tumor burden, especially if the lesions were located in dangerous sites (e.g., vertebral lesions at risk of compressing the spinal cord). Moreover, in contrast with the clinical trials, for patients treated as "compassionate use" or after the approval of the drug, there was no limit on prior therapy (i.e., treatment with other TKIs) and their ECOG score could be up to 2 . The main exclusion criteria were the presence of important cardiac disease or important biochemical alterations in blood samples before starting lenvatinib treatment. In our patients, lenvatinib treatment was withdrawn in the presence of progression disease according to the RECIST criteria or in the presence of severe AEs.

All patients gave their signed approval for the use of their personal data for research and scientific purposes as part of the policy of our University Hospital, and this study was approved by the local Ethics Committee (CEAVNO - Comitato Etico Area Vasta Nord Ovest). The study was conducted in accordance with the provisions of the Declaration of Helsinki (2013).

\section{Treatment and Follow-Up}

The clinical-pathological features and biochemical and morphological results of the patients were collected at the time of starting lenvatinib and during follow-up. The initial dose of lenvatinib was $24 \mathrm{mg}$ daily in all patients enrolled in the SELECT and EAP studies. In patients treated with lenvatinib as a "compassionate use" or after its approval, the starting dose was $24 \mathrm{mg} /$ day or lower (i.e., 20,14,10, or $8 \mathrm{mg}$ ) according to the patient's clinical condition. During follow-up, lenvatinib dose reduction or transient interruption was performed in cases of the appearance of severe AEs according to the Common Terminology Criteria of Adverse Events (CTCAE) [16].

Patients were evaluated at baseline and then according to the indications of the clinical trials. In patients treated with lenvatinib as "compassionate use" or as a commercial drug, the timing of follow-up was similar to that of patients enrolled in the clinical trials. The morphological tumor response was evaluated with a computed tomography (CT) scan during follow-up. Progressive disease (PD), 


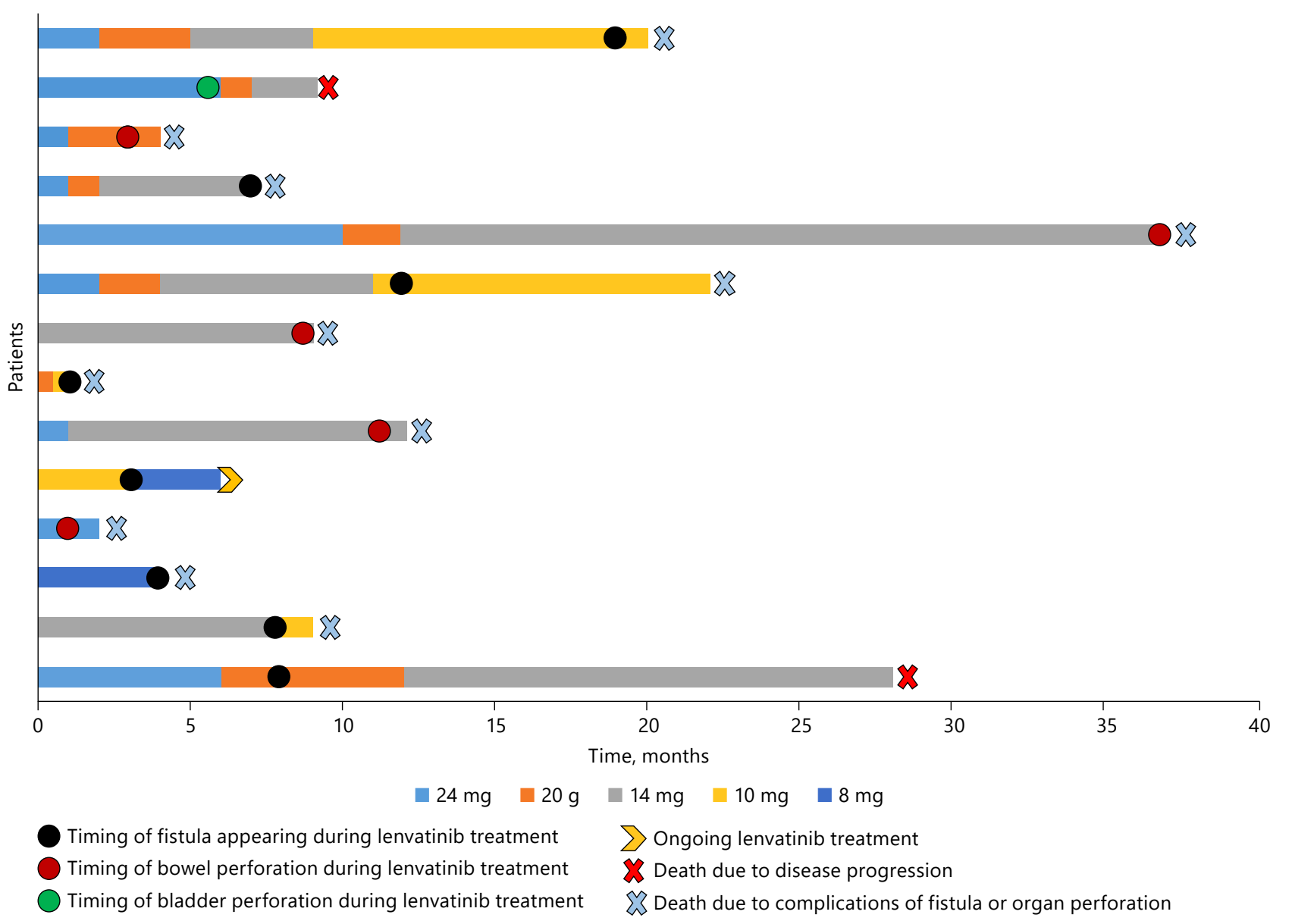

Fig. 1. Change of lenvatinib dose over the treatment period in patients with RAI-R DTC/PDTC who developed fistula and/or organ perforation. The changes in the dose intensity of lenvatinib are shown combined with the occurrence of the event of fistula and/ or organ perforation described as a hoop of a different color ac-

stable disease (SD), and a partial response (PR) were defined according to RECIST [15]. Tumor infiltration and the presence of fistula or organ perforation were evaluated by endoscopy and CT scans. We also evaluated the biochemical response (i.e., decreases in the serum levels of thyroglobulin [Tg] (Immulite 2000 Thyroglobulin; DPC, Los Angeles, CA, USA) and Tg antibodies [TgAb] (TOS, Tosoh A1S-600II; Tosoh Corp., San Francisco, CA, USA).

\section{Statistical Analysis}

Pearson's $\chi^{2}$ test and Fisher's exact test were used to evaluate the differences in counts and frequency between groups, as appropriate. Student's $t$-test and Mann-Whitney U test were used to assess differences between groups for continuous variables with Gaussian and skewed distributions, respectively. Data analysis was performed using StatView 4.5 software (Abacus Concepts Inc., Berkeley, CA, USA). $p<0.05$ was considered statistically significant. Data are presented as mean \pm SD or frequency (percentage).

Fistula/Organ Perforation on Lenvatinib Treatment cording to the site of the event (black hoop for the occurrence of fistula; red hoop for the occurrence of bowel perforation; green hoop for the occurrence of bladder perforation). RAI-R DTC, radioiodine-refractory differentiated thyroid cancer; PDTC, poorly differentiated thyroid cancer.

\section{Results}

Clinical-Pathological and Epidemiological Features of RAI-R DTC or PDTC Patients who Developed a Fistula or Organ Perforation during Lenvatinib Treatment

Between February 2011, when we enrolled the first patient in the SELECT study, and February 2020 (i.e., the data lock of the present study), a total of 95 locally advanced or metastatic RAI-R DTC or PDTC patients, referred to our center, started lenvatinib therapy. Fourteen of $95(14.7 \%)$ patients developed a fistula or organ perforation during lenvatinib treatment after a median followup of 7.5 months (mean: 9.1 months, range 1-37 months) 
Table 1. Correlation between epidemiological, pathological, and clinical features of patients who developed and not developed the fistula or organ perforation

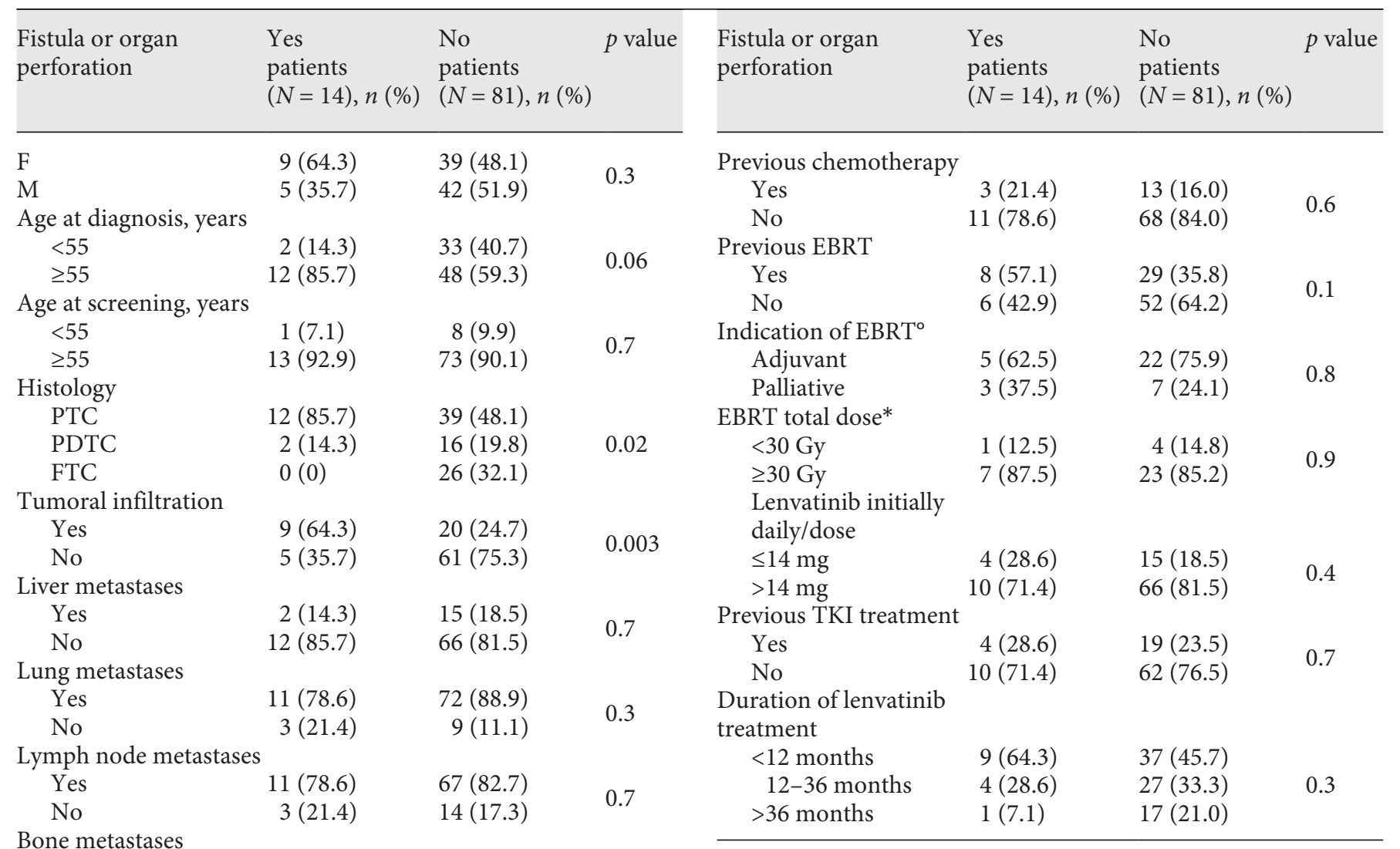

\begin{tabular}{llll} 
Bone metastases & & & \\
$\quad$ Yes & $5(35.7)$ & $39(48.1)$ & 0.4 \\
No & $9(64.3)$ & $42(51.9)$ & \\
$\begin{array}{l}\text { Previous re-surgery } \\
\text { Yes }\end{array}$ & $8(57.1)$ & $57(70.4)$ & \\
No & $6(42.9)$ & $24(29.6)$ & 0.3 \\
\hline
\end{tabular}

(Fig. 1). The epidemiological, clinical, and pathological features of these 14 patients are reported in Table 1. They were more frequently women with a median age of approximately 66 years at the time of starting lenvatinib. In the majority of cases, they were multimetastatic with a high prevalence of lymph node and lung metastases. More than $50 \%$ of the cases had been treated with external beam radiation therapy (EBRT), and almost $30 \%$ were previously treated with other TKIs.

Among cases who developed either fistula or organ perforation, 2 of $14(14.3 \%)$ patients were enrolled in the SELECT study, 2 of 14 (14.3\%) were enrolled in the EAP study, 4 of 14 (28.6\%) patients were treated with lenvatinib as "compassionate use," and 6 of 14 (42.8\%) patients were treated with the commercial drug. However, when considering the prevalence of these cases in the corresponding groups of patients enrolled in the same study (2/15 [13\%] and 2/8 [25\%] in the SELECT and EAP studies, respectively) or treated as "compassionate use" $(4 / 27[14.8 \%])$ or as a commercial drug $(6 / 45$ $[13.3 \%])$, there were no statistically significant differences.

Nine of 14 (64.3\%) patients started lenvatinib at a daily dose of $24 \mathrm{mg}, 1$ of 14 (7.1\%) patients started lenvatinib at daily dose of $20 \mathrm{mg}, 2$ of 14 (14.3\%) patients started lenvatinib at a daily dose of $14 \mathrm{mg}$, and 2 of $14(14.3 \%)$ started lenvatinib at a daily dose equal to or lower than $10 \mathrm{mg}$ ( 1 patient at $10 \mathrm{mg} /$ daily and 1 patient at $8 \mathrm{mg} /$ daily). As shown in Fig. 1, during follow-up, the lenvatinib daily dose was reduced in some patients due to the appearance of AEs. 

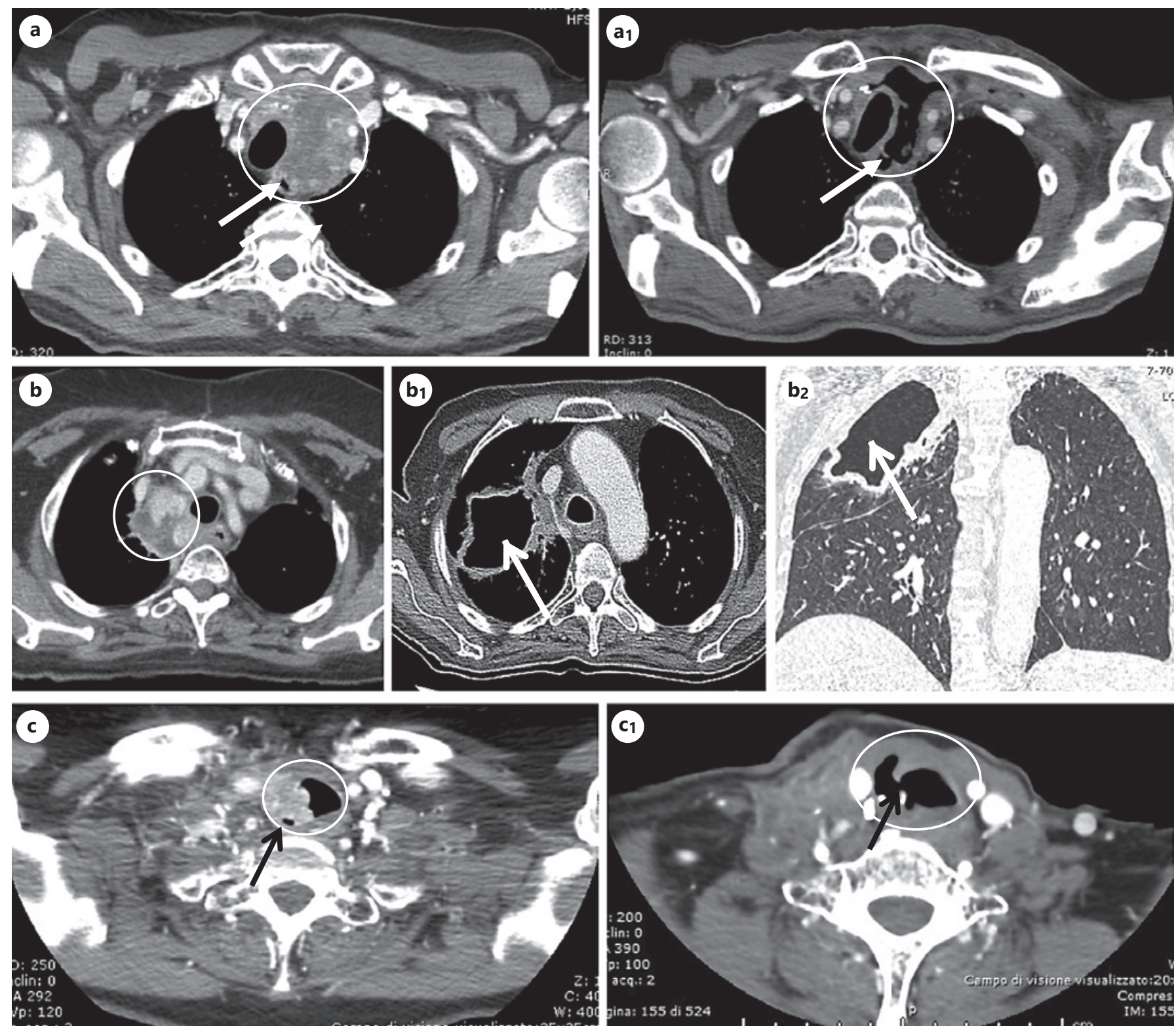

Fig. 2. CT images of some cases who developed fistula and/or organ perforation. a, a1 A large mediastinal lesion infiltrating the trachea (panel a) of a 68-year-old patient, which markedly shrank after 7 months of lenvatinib treatment, leading to tracheoesophageal fistula as indicated by the arrow (panel a 1). b, b1, b2 Multiple paratracheo-esophageal lymph node metastases infiltrating the right bronchial tree (panel b) of a 70-year-old patient; after 12 months of lenvatinib treatment, the patient developed a bronchial

fistula leading to a pulmonary cavitation of approximately $65 \mathrm{~cm}$ (panel b1: CT cross-sectional image of the pulmonary cavitation; panel $\mathrm{B}_{2}$ : CT coronal section image of the pulmonary cavitation). c, c1 A mediastinal lymph node lesion infiltrating the right tracheal wall of a 64-year-old patient (panel c), which markedly shrank after lenvatinib treatment, followed by the development of the tracheal perforation indicated by the arrow (panel c1). CT, computed tomography.

As shown in Table 2, 9 of $14(64.3 \%)$ patients had a tumor infiltration of the trachea, bronchus, esophagus, pleura, or even the bladder. With the exception of the case of bladder infiltration, which happened 6 months after starting the lenvatinib therapy, all of the other infiltra-

tions were already present at the time of starting the lenvatinib therapy. Five of 14 (35.7\%) had a bowel perforation, but only 2 had diverticulosis, as shown by CT scan, at the time of starting the lenvatinib therapy. Some examples of the fistulas are shown in Figure 2. 
Table 2. Tumoral infiltration sites and diverticulosis in patients treated with lenvatinib and developing fistulas or organ perforation

\begin{tabular}{lcc}
\hline & \multicolumn{2}{c}{ RAI-R DTC/PDTC patients } \\
\cline { 2 - 3 } & $n$ & $\%$ \\
\cline { 2 - 3 } & & \\
\hline Tumor infiltration site $(N=9)$ & 1 & 11.1 \\
$\quad$ Bronchus & 1 & 11.1 \\
Pleura & 4 & 44.5 \\
Trachea & 2 & 22.2 \\
Trachea-esophagus & 1 & 11.1 \\
Bladder & & \\
Fistula/perforation site & 1 & 7.1 \\
Bronchus & 1 & 7.1 \\
Pleura & 4 & 28.6 \\
Trachea & 2 & 14.3 \\
Trachea-esophagus & 1 & 7.1 \\
Bladder & 5 & \\
Bowel & & 14.3 \\
Diverticulosis & 2 & 85.7 \\
Yes & 12 & \\
No & &
\end{tabular}

RAI-R DTC, radioiodine-refractory differentiated thyroid cancer; PTDC, poorly differentiated thyroid cancer.

\section{Efficacy of Lenvatinib in Our Patients}

At the first CT scan evaluation, performed after 2 months of treatment for 13 of 14 patients, 4 of 13 (30.8\%) had a PR and 9 of 13 (69.2\%) showed SD. The best morphological response was a PR in 5 of $13(38.5 \%)$ patients and SD in 8 of $13(61.5 \%)$ patients.

At the last CT scan performed at the data cut-off (mean follow-up 12.1 months, median 9 months, range 1-37 months), 10 of 13 (76.9\%) patients showed SD and 3 of 13 (23.1\%) showed progressive disease. Thirteen of these 14 patients died a mean of 3 months (median 1 month, range 0.5-20 months) after the development of the fistula or organ perforation (11 of 13 [84.6\%]), while 2 of 13 (15.4\%) died due to disease progression. In all cases, lenvatinib was interrupted or reduced. In the other patient, lenvatinib treatment was interrupted after the appearance of the tracheal fistula (3 months after starting lenvatinib) and restarted at a lower daily dose, and she is still alive 6 months from the beginning of the therapy.

During the follow-up, we also observed a reduction in the Tg levels (median Tg at screening: $399.5 \mu \mathrm{g} / \mathrm{L}$ vs. median Tg at data cut-off: $76.6 \mu \mathrm{g} / \mathrm{L}$ ). In the patients with detectable Tg antibodies, they showed similar trends for Tg during the follow-up.
Safety and Tolerability of Lenvatinib in RAI-R DTC or PDTC Patients Who Developed Fistula or Organ Perforation during Treatment

Different types of AEs appeared in all patients, and the most frequent AEs were anorexia, asthenia, and weight loss. The AEs were mainly of grade 1 or 2 severity. The AEs reaching grade 3 or 4 in severity were mainly anorexia, weight loss, proteinuria, hypertension, and diarrhea. Ten patients developed high levels of thyroid-stimulating hormone and required an increase of their daily dose of levothyroxine. The other AEs were treated with symptomatic therapy and/or temporary interruption/reduction of the lenvatinib dose.

\section{Predictors of Fistula/Organ Perforation during \\ Lenvatinib Treatment}

The risk factor analysis performed between the 14 patients with fistula or organ perforation and the other patients who did not develop these severe AEs $(n=81)$ showed that the presence of tumor infiltration and the tumor histology (PTC and PDTC vs. FTC and Hurthle) were significantly correlated with the development of fistula or organ perforation $(p=0.003$ and $p=0.02$, respectively) (Table 1). Since at univariate analysis there were only 2 features statistically correlated with the fistula/organ perforation and this $\mathrm{AE}$ was present in only few cases, we could not perform the multivariate analysis, thus impairing the possibility to identify the independent prognostic factor for the development of fistula/organ perforation.

It is worth noting that despite the correlation between tumor infiltration and development of fistula, $24.7 \%$ of the cases had tumor infiltration but did not develop this severe AE (Table 1). When we analyzed the subgroup of patients with tumor infiltration at the time of lenvatinib therapy initiation (see online suppl. Table; see online Supplementary Materials), the only significant difference between those who developed fistulas or organ perforation and those who did not was the histological type (the PTC histotype) being the only potential predictor of fistula development. EBRT, its indication and total dose, the startingdose of lenvatinib, and the duration of treatment were apparently not relevant for the development of the fistula. Similarly, although both patients with diverticulosis had a bowel perforation during lenvatinib treatment, another 3 patients without any sign or symptoms of diverticulosis also developed this severe $\mathrm{AE}$. 


\section{Discussion}

It is known that lenvatinib can induce serious and lifethreatening AEs such as fistulas and organ perforations [9]. The mechanisms involved in the development of these serious AEs are poorly understood but are probably correlated with VEGFR inhibition as reported for the use of monoclonal VEGF antibody (bevacizumab) in the treatment of other solid tumors $[12,13]$. The main risk factors recognized as causes of fistula and/or organ perforation associated with anti-angiogenetic therapy in cancer patients are EBRT, tumor infiltration, recent endoscopy procedures, diverticulitis, and carcinomatosis $[4-6,8,12,17]$. Because of the importance of these AEs, the widespread use of lenvatinib in the treatment of RAIR DTC patients, and the limited information regarding these AEs, we analyzed the prevalence of fistulas and organ perforations in a large series of RAI-R DTC patients followed up at our center, and we tried to identify the possible predictors of developing these life-threatening complications.

In our series, we found that 14 of 95 (14.7\%) patients developed a fistula or organ perforation during lenvatinib treatment. Our incidence of fistula and/or organ perforation compared to that reported in the literature for the other anti-angiogenic drugs was quite high $[9,12,13]$. This result is probably due to faster and more potent anti-angiogenic action of lenvatinib treatment than that of the other TKIs.

Despite these AEs that, at the time of data cut-off, caused the death of $11 / 14$ patients, the radiological response evaluated using CT scanning performed after 2 months of lenvatinib treatment showed a PR in $30.8 \%$ of the patients and SD in $69.2 \%$ of the patients, confirming early disease control (i.e., PR + SD). Moreover, the median follow-up of these patients was approximately 9 months (mean: 12.1 months, range 1-37 months), and some of these cases survived for several months after the appearance of the fistula. Unfortunately, it is impossible to say if the treatment was able to prolong their survival time since we do not have a control arm.

Looking at the most frequent AEs in our series, we observed that the only life-threatening AE was fistula and/ or organ perforation; regarding the other common AEs, we found a similar profile to that reported in the SELECT study and in the other large series of RAI-R DTC patients treated with lenvatinib that have been published so far $[2$, 18]. The analysis of the possible predictors associated with the development of fistula and/or organ perforation showed that the presence of tumoral infiltration $(p=$

Fistula/Organ Perforation on Lenvatinib

Treatment
$0.003)$ and the tumor histology (PTC and PDTC) ( $p=$ 0.02 ) were significantly related to the occurrence of this complication. Although TKIs are much more cytostatic than cytotoxic, they are able to induce shrinkage of the tumoral mass as demonstrated by the relevant objective response obtained in the clinical trials $[2,3]$. This is particularly true for lenvatinib and, since it acts potently and rapidly, it is conceivable that when the infiltrating tumor mass is destroyed by the drug, the infiltrated tracheal or esophagus wall is destroyed too, causing the fistula. For this reason, cases with a local recurrence potentially infiltrating the trachea or esophagus should be always submitted to endoscopic evaluation of these organs to establish the level of infiltration.

Regarding the histology and the greater risk of having a fistula in PTC and PDTC than in FTC and Hurthle, this could be explained by the different biology of the different histotypes, with FTC more prone to metastasize at a distance than locally. However, this hypothesis should be confirmed in a larger series with more FTC cases.

Among the 5 patients with a bowel perforation, 2 patients had preexisting diverticulosis, the only 2 of the entire series, at the screening CT scanning. One patient was previously treated with EBRT to the hip bones while the other 2 did not show any obvious risk factors. Thus, although diverticulosis as well as EBRT can be considered risk factors, according to our findings, bowel perforation can happen independently from these conditions and cannot be easily predicted. Nevertheless, an accurate anamnestic evaluation of potential intestinal diseases should be always performed as well as an accurate evaluation of diverticulosis at the pre-therapeutic CT scan. Regarding EBRT, we observed that $87.5 \%$ of the patients who developed fistula/organ perforation were treated with a medium/higher dose of EBRT ( $\geq 30 \mathrm{~Gy}$ ), but at the same time approximately $35 \%$ of the patients who did not develop fistula were also treated with EBRT, and $85.2 \%$ of them were treated with $\geq 30 \mathrm{~Gy}$, suggesting that EBRT cannot be considered an exclusion criterion to start lenvatinib.

A limitation of the present study is its retrospective nature and the fact that data are from a single center. However, after the SELECT study, this is the study analyzing the biggest series of patients treated with lenvatinib. This allowed us to perform some statistical analysis about risk factors that can have an impact on current treatment of cases with organ infiltration such as the choice to treat or not to treat with EBRT and the starting dose of lenvatinib.

In conclusion, in metastatic thyroid cancer patients treated with lenvatinib, the presence of tumor infiltration 
and histological type (PTC or PDTC) should be considered as potential risk factors for the development of fistula or organ perforation, although they do not represent an absolute contraindication. These complications seem to be related to the anti-angiogenic effect of lenvatinib, which induces necrosis of the tumoral lesions independently of the starting dose. Despite EBRT and the presence of diverticulosis not being significantly associated with the development of fistula and organ perforation, a medium/ higher dose of EBRT, especially when focused on the tumor infiltration, and the presence of diverticulosis on a CT scan, should be regarded as potential additional reasons to develop this complication. Finally, according to our findings, there is no reason to start lenvatinib at a lower daily dose when tumor infiltration is present.

\section{Acknowledgement}

C.G. contributed to this article as recipient of the $\mathrm{PhD}$ program in clinical and translational sciences.

\section{Statement of Ethics}

All patients gave their signed approval for the use of their personal data for research and scientific purposes as part of the policy of our University Hospital, and this study was approved by the local Ethics Committee (CEAVNO - Comitato Etico Area Vasta Nord Ovest) (Reference number 57873). This study was conducted in accordance with the provisions of the Declaration of Helsinki (2013).

\section{Conflict of Interest Statement}

R.E. is a consultant for EISAI; however, the present study was not conditioned by this activity. L.V., C.G., L.A., E.M., D.V., V.B., A.M., L.P., L.L., V.C., and A.R. have nothing to disclose.

\section{Funding Sources}

This study has been supported by PRIN 2017: New insights into the molecular signature of differentiated thyroid cancer: implications for diagnosis, prognosis, and therapy (Prot. 2017YTWKWH).

\section{Author Contribution}

Laura Valerio and Carlotta Giani are the young endocrinologists who took care of the clinical management of the patients, collected and interpreted data, and were involved in the preparation and submission of the manuscript. Laura Agate, Eleonora Molinaro, David Viola, Valeria Bottici, Antonio Matrone, Luciana Puleo, Loredana Lorusso, and Virginia Cappagli are expert endocrinologists who took care of the clinical management of the patients and the organization of multidisciplinary meetings to discuss the clinical follow-up of the cases. Alessandro Ribechini is the endoscopist surgeon who took care of patients who developed gastrointestinal fistula. Rossella Elisei is the senior endocrinologist who was responsible of the clinical management of the patients and supervisor of manuscript preparation. All authors contributed to the study conception and design and approved the final manuscript.

\section{References}

1 Viola D, Valerio L, Molinaro E, Agate L, Bottici V, Biagini A, et al. Treatment of advanced thyroid cancer with targeted therapies: ten years of experience. Endocr Relat Cancer. 2016;23(4):R185-205.

2 Schlumberger M, Tahara M, Wirth LJ, Robinson B, Brose MS, Elisei R, et al. Lenvatinib versus placebo in radioiodine-refractory thyroid cancer. N Engl J Med. 2015;372(7):621-30.

3 Brose MS, Nutting CM, Jarzab B, Elisei R, Siena $\mathrm{S}$, Bastholt L, et al. Sorafenib in radioactive iodine-refractory, locally advanced or metastatic diff erentiated thyroid cancer: a randomised, double-blind, phase 3 trial. Lancet. 2014;384(9940):319-28.

4 Blevins DP, Dadu R, Hu M, Baik C, Balachandran D, Ross W, et al. Aerodigestive fistula formation as a rare side effect of antiangiogenic tyrosine kinase inhibitor therapy for thyroid cancer. Thyroid. 2014; 24(5):918-22.

5 Tori M, Shimo T. Long-term efficacy of lenvatinib for recurrent papillary thyroid carcinoma after multimodal treatment and management of complications: a case report. BMC Cancer. 2018;18(1):698.

6 Britt CJ, Russell JO. Tyrosine kinase inhibitor use and wound healing in tracheoesophageal punctures. Ear Nose Throat J. 2019;98(8): 510-2.

7 Date E, Okamoto K, Fumita S, Kaneda H. Gastrointestinal perforation related to lenvatinib, an anti-angiogenic inhibitor that targets multiple receptor tyrosine kinases, in a patient with metastatic thyroid cancer. Invest New Drugs. 2018;36(2):350-3.
8 Staub Y, Nishiyama A, Suga Y, Fujita M, Matsushita R, Yano S. Clinical characteristics associated with lenvatinib-induced fistula and tumor-related bleeding in patients with thyroid cancer. Anticancer Res. 2019;39(7): 3871-8.

9 Costa R, Carneiro BA, Chandra S, Pai SG, Chae YK, Kaplan JB, et al. Spotlight on lenvatinib in the treatment of thyroid cancer: patient selection and perspectives. Drug Des Devel Ther. 2016;10:873-84.

10 Matrone A, Valerio L, Pieruzzi L, Giani C, Cappagli V, Lorusso L, et al. Protein kinase inhibitors for the treatment of advanced and progressive radiorefractory thyroid tumors: from the clinical trials to the real life. Best Pract Res Clin Endocrinol Metab. 2017;31(3): 319-34. 
11 Cabanillas ME, Takahashi S. Managing the adverse events associated with lenvatinib therapy in radioiodine-refractory differentiated thyroid cancer. Semin Oncol. 2019;46(1): 57-64.

12 Walraven M, Witteveen PO, Lolkema MP, Van Hillegersberg R, Voest EE, Verheul HM. Antiangiogenic tyrosine kinase inhibition related gastrointestinal perforations: a case report and literature review. Angiogenesis. 2011;14(2):135-41.

13 Keating GM. Bevacizumab: a review of its use in advanced cancer. Drugs. 2014;74(16): 1891-925.
14 Giani C, Valerio L, Bongiovanni A, Durante C, Grani G, Ibrahim T, et al. Safety and quality of life data from an Italian expanded access program of lenvatinib for treatment of thyroid cancer. Thyroid. 2020 Sep 10. Online ahead of print.

15 Eisenhauer EA, Therasse P, Bogaerts J, Schwartz LH, Sargent D, Ford R, et al. New response evaluation criteria in solid tumours: Revised RECIST guideline (version 1.1). Eur J Cancer. 2009;45(2):228-47.
16 National Institute of Cancer. Common terminology criteria for adverse events (CTCAE), version 4.0, DCTD, CTI, NIH. Victoria: DHHS; 2009.

17 Hoshino Y, Hasegawa H, Ishii Y, Endo T, Ochiai $\mathrm{H}$, Okabayashi $\mathrm{K}$, et al. Two cases of bowel perforation associated with sunitinib treatment for renal cell carcinoma. Int J Clin Oncol. 2012;17(4):412-6.

18 Berdelou A, Borget I, Godbert Y, Nguyen T, Garcia M-E, Chougnet CN, et al. Lenvatinib for the treatment of radioiodine-refractory thyroid cancer in real-life practice. Thyroid. 2018;28(1) 